\title{
Certain results on (p,q)-Hermite based Apostol type Frobenius-Euler polynomials
}

\author{
Waseem Ahmad Khan ${ }^{1}$, Idrees Ahmad Khan ${ }^{2}$, Ugur Duran ${ }^{3, *}$ and Mehmet Acikgoz ${ }^{4}$ \\ ${ }^{1}$ Department of Mathematics, Faculty of Science, \\ Integral University, Lucknow-226026, India \\ E-Mail: waseem08 khan@rediffmail.com \\ ${ }^{2}$ Department of Mathematics, Faculty of Science, \\ Integral University, Lucknow-226026, India \\ E-Mail: khanidrees077@gmail.com \\ ${ }^{3}$ Department of the Basic Concepts of Engineering, \\ Faculty of Engineering and Natural Sciences, \\ Iskenderun Technical University, TR-31200 Hatay, Turkey \\ E-Mail: mtdrnugur@gmail.com \& ugur.duran@iste.edu.tr \\ ${ }^{*}$ Corresponding Author \\ ${ }^{4}$ University of Gaziantep, Faculty of Science and Arts, \\ Department of Mathematics, TR-27310 Gaziantep, Turkey \\ E-Mail: acikgoz@gantep.edu.tr
}

\begin{abstract}
In the present paper, the $(p, q)$-Hermite based Apostol type Frobenius-Euler polynomials and numbers are firstly considered and then diverse basic identities and properties for the mentioned polynomials and numbers, including addition theorems, difference equations, integral representations, derivative properties, recurrence relations. Moreover, we provide summation formulas and relations associated with the Stirling numbers of the second kind.
\end{abstract}

2010 Mathematics Subject Classification. Primary 05A13, Secondary 11B68, 11B73, 33C45.

Key Words and Phrases. Hermite polynomials, Apostol type Frobenius-Euler polynomials, Hermite based Apostol type Frobenius Euler polynomials, Stirling numbers of the second kind, $(p, q)$-numbers.

\section{INTRODUCTION}

The $(p, q)$-numbers are defined as:

$$
[n]_{p, q}=p^{n-1}+p^{n-2} q+p^{n-3} q^{2}+\cdots+p q^{n-2}+q^{n-1}=\frac{p^{n}-q^{n}}{p-q} .
$$

We can write easily that $[n]_{p, q}=p^{n-1}[n]_{q / p}$, where $[n]_{q / p}$ is the $q$-number in $q$-calculus given by $[n]_{q / p}=$ $\frac{(q / p)^{n}-1}{(q / p)-1}$. Thereby, this implies that $(p, q)$-numbers and $q$-numbers are different, that is, we can not obtain $(p, q)$-numbers just by substituting $q$ by $q / p$ in the definition of $q$-numbers. However, in the case of $p=1$, $(p, q)$-numbers reduce to $q$-numbers, (see[3-8]). 
The $(p, q)$-derivative of a function $f$ with respect to $x$ is defined by

$$
D_{p, q} f(x)=D_{p, q} f(x)=\frac{f(p x)-f(q x)}{(p-q) x},(x \neq 0)
$$

and $D_{p, q} f(0)=f^{\prime}(0)$, provided that $f$ is differentiable at 0 . The $(p, q)$-derivative operator holds the following properties

and

$$
D_{p, q}(f(x) g(x))=g(p(x)) D_{p, q} f(x)+f(q x) D_{p, q} g(x),
$$

$$
D_{p, q}\left(\frac{f(x)}{g(x)}\right)=\frac{g(q x) D_{p, q} f(x)-f(q x) D_{p, q} g(x)}{g(p x) g(q x)} .
$$

The $(p, q)$-analogue of $(x+a)^{n}$ is given by

$$
\begin{gathered}
(x+a)_{p, q}^{n}=(x+a)(p x+a q) \cdots\left(p^{n-2} x+a q^{n-2}\right)\left(p^{n-1} x+a q^{n-1}\right), n \geq 1 \\
=\sum_{k=0}^{n}\left(\begin{array}{l}
n \\
k
\end{array}\right)_{p, q} p^{\left(\begin{array}{c}
n \\
2
\end{array}\right)} q^{\left(\begin{array}{c}
n-k \\
2
\end{array}\right)} x^{k} a^{n-k},
\end{gathered}
$$

where the $(p, q)$-Gauss binomial coefficients $\left(\begin{array}{l}n \\ k\end{array}\right)_{p, q}$ and $(p, q)$-factorial $[n]_{p, q}$ ! are defined by

$$
\left(\begin{array}{l}
n \\
k
\end{array}\right)_{p, q}=\frac{[n]_{p, q} !}{[n-k]_{p, q ![k]_{p, q} !}}(n \geq k) \text { and }[n]_{p, q} !=[n]_{p, q} \cdots[2]_{p, q}[1]_{p, q}, \quad(n \in \mathbb{N}) .
$$

The two types of the $(p, q)$-exponential functions given by

$$
e_{p, q}(x)=\sum_{n=0}^{\infty} \frac{p^{\left(\begin{array}{c}
n \\
2
\end{array}\right)} x^{n}}{[n]_{p, q} !} \text { and } E_{p, q}(x)=\sum_{n=0}^{\infty} \frac{q^{\left(\begin{array}{c}
n \\
2
\end{array}\right)} x^{n}}{[n]_{p, q} !}
$$

hold the following identities

$$
e_{p, q}(x) E_{p, q}(-x)=1 \text { and } e_{p^{-} q^{-}}(x)=E_{p, q}(x),
$$

and have the following $(p, q)$-derivative properties

$$
D_{p, q} e_{p, q}(x)=e_{p, q}(p x) \text { and } D_{p, q} E_{p, q}(x)=E_{p, q}(q x) .
$$

The definite $(p, q)$-integral is given by

in conjunction with

$$
\int_{0}^{a} f(x) d_{p, q} x=(p-q) a \sum_{k=0}^{\infty} \frac{p^{k}}{q^{k+1}} f\left(a \frac{p^{k}}{q^{k+1}}\right),
$$

$$
\left.\int_{a}^{b} f(x) d_{p, q} x=\int_{0}^{b} f(x) d_{p, q} x-\int_{0}^{a} f(x) d_{p, q} x, \quad \text { (see }[20]\right) .
$$

Recently, $(p, q)$-generalizations of some special polynomials have been introduced and investigated by several mathematicians, $c f$. $[23,5-8,21]$ and see the references cited therein. Duran et al. [8] introduced a new kind of Bernoulli, Euler and Genocchi polynomials based on the $(p, q)$-numbers and developed their some properties involving addition theorems, difference equations, derivative properties, recurrence relationships including $(p, q)$-extensions of the Cheon's main result [2] and the formula of Srivastava and Pintér [22]. Duran et al. $[6,7]$ considered $(p, q)$-generalizations of the Apostol-Bernoulli, Apostol-Euler, Apostol-Genocchi and Apostol Frobenius-Euler polynomials and derived their identities and properties. Duran et al. [5] defined $(p, q)$-Frobenius-Euler numbers and polynomials and researched their some identities and properties including addition property, difference equation, derivative property, recurrence relationships including integral representation, explicit formulae and relations for these polynomials and numbers. Moreover, they derived some correlations for $(p, q)$-Frobenius-Euler polynomials of order $\alpha$ associated with $(p, q)$-Bernoulli polynomials, $(p, q)$-Euler polynomials and $(p, q)$-Genocchi polynomials in [5]. Sadjang [20] introduced polynomial sets of $(p, q)$-Appell type polynomials and provided some of their characterizations and the algebraic properties of the set of all polynomial sequences of $(p, q)$-Appell type a recurrence relation and a $(p, q)$-difference equation 
for those polynomials. He also attained a set of $(p, q)$-Hermite polynomials with their three-term recurrence relation and a second order homogeneous $(p, q)$-difference equation in [21].

The generalized $(p, q)$-Bernoulli polynomials, the generalized $(p, q)$-Euler polynomials and the generalized $(p, q)$-Genocchi polynomials are defined by means of the following generating function as follows (see $[1,3-$ $10,20,21])$ :

$$
\begin{aligned}
& \left(\frac{t}{e_{p, q}(t)-1}\right)^{\alpha} e_{p, q}(x t)=\sum_{n=0}^{\infty} B_{n}^{(\alpha)}(x: p, q) \frac{t^{n}}{[n]_{p, q} !},|t|<2 \pi \\
& \left(\frac{2}{e_{p, q}(t)+1}\right)^{\alpha} e_{p, q}(x t)=\sum_{n=0}^{\infty} E_{n}^{(\alpha)}(x: p, q) \frac{t^{n}}{[n]_{p, q} !},|t|<\pi
\end{aligned}
$$

and

$$
\left(\frac{2 t}{e_{p, q}(t)+1}\right)^{\alpha} e_{p, q}(x t)=\sum_{n=0}^{\infty} G_{n}^{(\alpha)}(x: p, q) \frac{t^{n}}{[n]_{p, q} !},|t|<\pi
$$

The corresponding numbers of the mentioned polynomials are given as follows:

$$
B_{n}^{(\alpha)}(0: p, q):=B_{n}^{(\alpha)}(p, q), E_{n}^{(\alpha)}(0: p, q):=E_{n}^{(\alpha)}(p, q)
$$

and

$$
G_{n}^{(\alpha)}(0: p, q):=G_{n}^{(\alpha)}(p, q)(n \in \mathbb{N})
$$

Duran et al. [7] defined Apostol type $(p, q)$-Bernoulli polynomials $B_{n}^{(\alpha)}(x, y ; \lambda: p, q)$ of order $\alpha$, the Apostol type $(p, q)$-Euler polynomials $E_{n}^{(\alpha)}(x, y ; \lambda: p, q)$ of order $\alpha$ and the Apostol type $(p, q)$-Genocchi polynomials $G_{n}^{(\alpha)}(x, y ; \lambda: p, q)$ of order $\alpha$ are defined by means of the following generating functions:

$$
\begin{aligned}
& \left(\frac{t}{\lambda e_{p, q}(t)-1}\right)^{\alpha} e_{p, q}(x) t E_{p, q} y t=\sum_{n=0}^{\infty} B_{n}^{(\alpha)}(x, y, \lambda: p, q) \frac{t^{n}}{[n]_{p, q} !},(|t+\log \lambda|<2 \pi), \\
& \left(\frac{2}{\lambda e_{p, q}(t)+1}\right)^{\alpha} e_{p, q}(x t) E_{p, q} y t=\sum_{n=0}^{\infty} E_{n}^{(\alpha)}(x, y, \lambda: p, q) \frac{t^{n}}{[n]_{p, q} !},(|t+\log \lambda|<\pi),
\end{aligned}
$$

and

$$
\left(\frac{2 t}{\lambda e_{p, q}(t)+1}\right)^{\alpha} e_{p, q}(x) t E_{p, q} y t=\sum_{n=0}^{\infty} G_{n}^{(\alpha)}(x, y, \lambda: p, q) \frac{t^{n}}{[n]_{p, q} !}, \quad(|t+\log \lambda|<\pi) .
$$

When $x=y=0$, one can get the corresponding numbers of the given polynomials above as follows:

$$
B_{n}^{(\alpha)}(0,0, \lambda: p, q):=B_{n}^{(\alpha)}(\lambda: p, q), E_{n}^{(\alpha)}(0,0, \lambda: p, q):=E_{n}^{(\alpha)}(\lambda: p, q)
$$

and

$$
G_{n}^{(\alpha)}(0,0, \lambda: p, q):=G_{n}^{(\alpha)}(\lambda: p, q)(n \in \mathbb{N})
$$

where $B_{n}^{(\alpha)}(\lambda: p, q), E_{n}^{(\alpha)}(\lambda: p, q)$ and $G_{n}^{(\alpha)}(\lambda: p, q)$ denote Apostol type $(p, q)$-Bernoulli numbers of order $\alpha$, the Apostol type $(p, q)$-Euler numbers of order $\alpha$ and the Apostol type $(p, q)$-Genocchi numbers of order $\alpha$.

Recently, Duran and Acikgoz [6] introduced and studied Apostol type $(p, q)$-Frobenius-Euler polynomials $H_{n}^{(\alpha)}(x, y ; u ; \lambda: p, q)$ defined as follows.

Definition 1.1. The generalized Apostol type $(p, q)$-Frobenius-Euler polynomials $H_{n}^{(\alpha)}(x, y ; u ; \lambda: p, q)$ of order $\alpha$ are defined by means of the following generating function:

$$
\left(\frac{1-u}{\lambda e_{p, q}(t)-u}\right)^{\alpha} e_{p, q}(x t) E_{p, q} y t=\sum_{n=0}^{\infty} H_{n}^{(\alpha)}(x, y ; u ; \lambda: p, q) \frac{t^{n}}{[n]_{p, q} !},
$$

where $\alpha$ and $\lambda$ is suitable (real or complex) parameters, $p, q \in \mathbb{C}$ with $0<|q|<|p| \leq 1$ and $u \in \mathbb{C} /\{1\}$. 
Remark 1.1. For $x=y=0$ and $\alpha=1$ in (2.1), the result reduces to

$$
\left(\frac{1-u}{\lambda e_{p, q}(t)-u}\right)=\sum_{n=0}^{\infty} H_{n}(u ; \lambda: p, q) \frac{t^{n}}{[n]_{p, q} !},
$$

where $H_{n}(u ; \lambda: p, q)$ denotes the Apostol type $(p, q)$-Frobenius-Euler numbers.

From (1.15), we have

$$
\begin{array}{cl}
H_{n}^{(1)}(x, y ; u ; \lambda: p, q):=H_{n}(x, y ; u ; \lambda: p, q) \\
\left.H_{n}^{(\alpha)}(x, y ; u ; \lambda: p, q)\right|_{p=1}:=H_{n, q}^{(\alpha)}(x, y ; u ; \lambda), \quad(\text { see }[9,18]) \\
\lim _{q \rightarrow p=1} H_{n}^{(\alpha)}(x, y ; u ; \lambda: p, q):=H_{n}^{(\alpha)}(x+y ; u ; \lambda), \quad(\text { see }[9,10,16]) .
\end{array}
$$

Note that

$$
H_{n}^{(0)}(x, y ; u ; \lambda: p, q)=\sum_{k=0}^{n}\left(\begin{array}{l}
n \\
k
\end{array}\right)_{p, q} p^{\left(\begin{array}{c}
k \\
2
\end{array}\right)} q^{\left(\begin{array}{c}
n-k \\
2
\end{array}\right)} x^{k} y^{n-k}=(x+y)_{p, q}^{n} .
$$

Very recently, Sadjang [21] introduced a new type of the $(p, q)$-Hermite polynomials, which is defined by means of the following generating function:

$$
F_{p, q}(x, t)=F_{p, q}(t) e_{p, q}(x t)=e_{p, q}\left(x t-\frac{t^{2}}{2}\right)=\sum_{n=0}^{\infty} H_{n}(x: p, q) \frac{t^{n}}{[n]_{p, q} !} .
$$

An explicit representation of the $(p, q)$-Hermite polynomials is obtained:

$$
H_{n}(x: p, q)=\sum_{k=0}^{\left[\frac{n}{2}\right]} \frac{(-1)^{k} p^{\left(\begin{array}{c}
n-2 k \\
2
\end{array}\right)+k(k-1)}[n]_{p, q} !}{[2 k]_{p, q} ! ![n-2 k]_{p, q} !} x^{n-2 k} .
$$

In this paper, we introduce the $(p, q)$-Hermite based Apostol type Frobenius-Euler numbers and polynomials and rthen develop some basic identities and properties. We also establish some useful and interesting relations and formulas for the mentioned polynomials and numbers. Moreover, we derive multifarious relationships for the $(p, q)$-Hermite based Apostol type Frobenius-Euler polynomials of order $\alpha$ related to the some known polynomials such as the $(p, q)$-Apostol type Bernoulli polynomials, the $(p, q)$-Apostol type Euler polynomials and the $(p, q)$-Apostol type Genocchi polynomials. Further, we derive summation formulas covering the Stirling numbers of the second kind.

2. The $(p, q)$-Hermite Based Apostol type Frobenius-Euler polynomials ${ }_{H} H_{n}^{(\alpha, s)}(x, y ; u ; \lambda: p, q)$

In this section, we introduce $(p, q)$-Hermite based Apostol type Frobenius-Euler polynomials $((p, q)$ HbAtFEp ${ }_{H} H_{n}^{(\alpha, s)}(x, y ; u ; \lambda: p, q)$ by means of the generating function and series representation. Certain relations for these polynomials are also derived by using various identities. Now we start at the following definition.

Definition 2.1. Let $p, q \in \mathbb{C}, \lambda \in \mathbb{C}, \alpha \in \mathbb{N}, 0<|q|<|p| \leq 1$. The generalized $(p, q)$-Hermite based Apostol type Frobenius-Euler polynomials ${ }_{H} H_{n}^{(\alpha, s)}(x, y ; u ; \lambda: p, q)$ of order $\alpha$ are defined by means of the following generating function:

$$
\left(\frac{1-u}{\lambda e_{p, q}(t)-u}\right)^{\alpha} e_{p, q}\left(x t-\frac{s t^{2}}{2}\right) E_{p, q}(y t)=\sum_{n=0}^{\infty}{ }_{H} H_{n}^{(\alpha, s)}(x, y ; u ; \lambda: p, q) \frac{t^{n}}{[n]_{p, q} !} .
$$

When $x=y=s=0$ in $(2.1),{ }_{H} H_{n}^{(\alpha, 0)}(0,0 ; u ; \lambda: p, q)=H_{n}^{(\alpha)}(u ; \lambda: p, q)$ are called the Apostol type $(p, q)$-Frobenius-Euler numbers of order $\alpha,(c f .[6])$. 
Remark 2.1. For $x=y=0$ in $(2.1),{ }_{H} H_{n}^{(\alpha, s)}(u ; \lambda: p, q)={ }_{H} H_{n}^{(\alpha, s)}(0,0 ; u ; \lambda: p, q)$ are called the $(p, q)$ Hermite based Apostol type Frobenius-Euler numbers defined by

$$
\left(\frac{1-u}{\lambda e_{p, q}(t)-u}\right)^{\alpha} e_{p, q}\left(\frac{-s t^{2}}{2}\right)=\sum_{n=0}^{\infty}{ }_{H} H_{n}^{(\alpha, s)}(u ; \lambda: p, q) \frac{t^{n}}{[n]_{p, q} !} .
$$

Remark 2.2. On setting $\alpha=0,(2.1)$ reduces to

$$
e_{p, q}\left(x t-\frac{s t^{2}}{2}\right) E_{p, q}(y t)=\sum_{n=0}^{\infty} H_{n}^{(s)}(x, y: p, q) \frac{t^{n}}{[n]_{p, q} !} .
$$

Theorem 2.1. The following series representation for the $(p, q)$-Hermite based Apostol type Frobenius-Euler polynomials ${ }_{H} H_{n}^{(\alpha, s)}(x, y ; u ; \lambda: p, q)$ of order $\alpha$ holds true:

$$
{ }_{H} H_{n}^{(\alpha, s)}(x, y ; u ; \lambda: p, q)=\sum_{m=0}^{n}\left(\begin{array}{c}
n \\
m
\end{array}\right)_{p, q} H_{n-m}^{(\alpha)}(y ; u ; \lambda: p, q)(\lambda) H_{m}^{(s)}(x: p, q) .
$$

Proof. Using equation (1.15) and (1.17) in the left hand side of equation (2.1) and then applying the Cauchy product rule and equating the coefficients of same powers of $t$ in both sides of resultant equation, we get representation (2.4).

Theorem 2.2. The following summation formula for the $(p, q)$-HbAtFEp holds true:

$$
\begin{aligned}
{ }_{H} H_{n}^{(\alpha, s)}(x, y ; u ; \lambda: p, q) & =\sum_{m=0}^{n}\left(\begin{array}{c}
n \\
m
\end{array}\right)_{p, q}{ }_{H} H_{m}^{(\alpha, s)}(0,0 ; u ; \lambda: p, q)(x+y)_{p, q}^{n-m} . \\
{ }_{H} H_{n}^{(\alpha, s)}(x, y ; u ; \lambda: p, q) & =\sum_{m=0}^{n}\left(\begin{array}{c}
n \\
m
\end{array}\right)_{p, q}{ }_{H} H_{m}^{(\alpha, s)}(0, y ; u ; \lambda: p, q) p^{\left(\begin{array}{c}
n-m \\
2
\end{array}\right)} x^{n-m} . \\
{ }_{H} H_{n}^{(\alpha, s)}(x, y ; u ; \lambda: p, q) & =\sum_{m=0}^{n}\left(\begin{array}{c}
n \\
m
\end{array}\right)_{p, q}{ }_{H} H_{m}^{(\alpha, s)}(x, 0 ; u ; \lambda: p, q) q^{\left(\begin{array}{c}
n-m \\
2
\end{array}\right)} y^{n-m} .
\end{aligned}
$$

Proof. Suitably using equations (1.4)-(1.6) in generating function (2.1) to get three different form. Further making use of the Cauchy product rule in the resultant expressions and then comparing the like powers of $t$ in the both sides of resultant equation, we find formulas (2.5)-(2.7).

Theorem 2.3. The following recursive formulas for the $(p, q)$-Hermite based Apostol type Frobenius-Euler polynomials ${ }_{H} H_{n}^{(\alpha, s)}(x, y ; u ; \lambda: p, q)$ of order $\alpha$ holds true:

$$
D_{p, q ; x H} H_{n}^{(\alpha, s)}(x, y ; u ; \lambda: p, q)=[n]_{p, q H} H_{n-1}^{(\alpha, s)}(p x, y ; u ; \lambda: p, q)
$$

and

$$
D_{p, q ; y H} H_{n}^{(\alpha, s)}(x, y ; u ; \lambda: p, q)=[n]_{p, q H} H_{n-1}^{(\alpha, s)}(x, q y ; u ; \lambda: p, q) .
$$

Proof. Differentiating generating function (2.1) with respect to $x$ and $y$ with the help of equation (1.2) and then simplifying with the help of the Cauchy product rule formulas (2.8) and (2.9) are obtained.

Theorem 2.4. The following difference formulas for the $(p, q)$-Hermite based Apostol type Frobenius-Euler polynomials ${ }_{H} H_{n}^{(\alpha, s)}(x, y ; u ; \lambda: p, q)$ of order $\alpha$ holds true:

$$
\begin{gathered}
\lambda_{H} H_{n}^{(\alpha, s)}(1, y ; u ; \lambda: p, q)-u_{H} H_{n}^{(\alpha, s)}(0, y ; u ; \lambda: p, q) \\
=(1-u)_{H} H_{n}^{(\alpha-1, s)}(0, y ; u ; \lambda: p, q) \\
\lambda_{H} H_{n}^{(\alpha, s)}(x, 0 ; u ; \lambda: p, q)-u_{H} H_{n}^{(\alpha, s)}(x,-1 ; u ; \lambda: p, q) \\
=(1-u)_{H} H_{n}^{(\alpha-1, s)}(x,-1 ; u ; \lambda: p, q) .
\end{gathered}
$$

Proof. By using definition (2.1), we can easily proof of equations (2.10) and (2.11). We omit the proof. 
Theorem 2.5. Let $\alpha, \beta \in \mathbb{N}$, the $(p, q)$-Hermite based Apostol type Frobenius-Euler polynomials ${ }_{H} H_{n}^{(\alpha, s)}(x, y ; u ; \lambda$ : $p, q)$ of order $\alpha$ holds true:

$$
\begin{gathered}
H_{n} H_{n}^{(\alpha+\beta, s)}(x, y ; u ; \lambda: p, q) \\
=\sum_{m=0}^{n}\left(\begin{array}{c}
n \\
m
\end{array}\right)_{p, q}{ }_{H} H_{n-m}^{(\alpha, s)}(0,0 ; u ; \lambda: p, q) H_{m}^{(\beta)}(x, y ; u ; \lambda: p, q), \\
\frac{u_{H} H_{n}^{(s)}(x, y ; u ; \lambda: p, q)}{(u-1)} \\
=\frac{\lambda}{u-1} \sum_{m=0}^{n}\left(\begin{array}{c}
n \\
m
\end{array}\right)_{p, q} p_{H}^{\left(\begin{array}{c}
m \\
2
\end{array}\right)} H_{n-m}^{(s)}(0,0 ; u ; \lambda: p, q)+H_{n}(x, y ; u ; \lambda: p, q), \\
=\sum_{m=0}^{n}\left(\begin{array}{c}
n \\
m
\end{array}\right)_{p, q} H_{n}^{(\alpha-\beta, s)}(x, y ; u ; \lambda: p, q) \\
H_{n-m}^{(\alpha, s)}(0,0 ; u ; \lambda: p, q) H_{m}^{(-\beta)}(x, y ; u ; \lambda: p, q),
\end{gathered}
$$

Proof. Using generating function (2.1), we get the equations (2.12)-(2.13). We omit the proof.

\section{Main results}

In this section, we establish some results on $(p, q)$-Hermite based Apostol type Frobenius-Euler polynomials ${ }_{H} H_{n}^{(\alpha, s)}(x, y ; u ; \lambda: p, q)$ of order $\alpha$ and some relationships for $(p, q)$-Hermite based Apostol type Frobenius-Euler polynomials of order $\alpha$ related to the $(p, q)$-Apostol type Bernoulli polynomials, the $(p, q)$ Apostol type Euler polynomials and the $(p, q)$-Apostol type Genocchi polynomials. We now begin with the following theorem.

Theorem 3.1. The following relation for the $(p, q)$-Hermite based Apostol type Frobenius-Euler polynomials ${ }_{H} H_{n}^{(\alpha, s)}(x, y ; u ; \lambda: p, q)$ of order $\alpha$ holds true:

Proof. We set

$$
\begin{gathered}
(2 u-1) \sum_{k=0}^{n}\left(\begin{array}{l}
n \\
k
\end{array}\right)_{p, q} H_{k}(0, y ; u ; \lambda: p, q)_{H} H_{n-k}^{(s)}(x, 0 ; 1-u ; \lambda: p, q) \\
=u_{H} H_{n}^{(s)}(x, y ; u ; \lambda: p, q)-(1-u)_{H} H_{n}^{(s)}(x, y ; 1-u ; \lambda ; p, q) .
\end{gathered}
$$

$$
\frac{(2 u-1)}{\left(\lambda e_{p, q}(t)-u\right)\left(\lambda e_{p, q}(t)-(1-u)\right)}=\frac{1}{\lambda e_{p, q}(t)-u}-\frac{1}{\lambda e_{p, q}(t)-(1-u)} .
$$

From the above equation, we see that

$$
\begin{gathered}
(2 u-1) \frac{(1-u) e_{p, q}\left(x t-\frac{s t^{2}}{2}\right)(1-(1-u)) E_{p, q}(y t)}{\left(\lambda e_{p, q}(t)-u\right)\left(\lambda e_{p, q}(t)-(1-u)\right)} \\
=\frac{(1-u) e_{p, q}\left(x t-\frac{s t^{2}}{2}\right) u E_{p, q}(y t)}{\lambda e_{p, q}(t)-u}-\frac{(1-u) e_{p, q}\left(x t-\frac{s t^{2}}{2}\right) E_{p, q}(y t)(1-(1-u))}{\lambda e_{p, q}(t)-(1-u)},
\end{gathered}
$$

which on using equations (1.15) and (2.1) in both sides, we have

$$
\begin{aligned}
& (2 u-1)\left(\sum_{k=0}^{\infty} H_{k}(0, y ; u ; \lambda: p, q) \frac{t^{k}}{[k]_{p, q} !}\right)\left(\sum_{n=0}^{\infty} H_{n}^{(s)}(x, 0 ; 1-u ; \lambda: p, q) \frac{t^{n}}{[n]_{p, q} !}\right) \\
= & u \sum_{n=0}^{\infty} H_{n}^{(s)}(x, y ; u ; \lambda: p, q) \frac{t^{n}}{[n]_{p, q} !}-(1-u) \sum_{n=0}^{\infty} H_{n}^{(s)}(x, y ; 1-u ; \lambda: p, q) \frac{t^{n}}{[n]_{p, q} !} .
\end{aligned}
$$


Applying the Cauchy product rule in the above equation and then equating the coefficients of like powers of $t$ in both sides of the resultant equation, assertion (3.1) follows.

Theorem 3.2. The following relation for the $(p, q)$-Hermite based Apostol type Frobenius-Euler polynomials ${ }_{H} H_{n}^{(\alpha, s)}(x, y ; u ; \lambda: p, q)$ of order $\alpha$ holds true:

$$
\begin{gathered}
u_{H} H_{n}^{(s)}(x, y ; u ; \lambda: p, q)=\sum_{k=0}^{n}\left(\begin{array}{l}
n \\
k
\end{array}\right)_{p, q} \\
\times\left[\lambda_{H} H_{n}^{(s)}(x, y ; u ; \lambda: p, q) p^{\left(\begin{array}{c}
n-k \\
2
\end{array}\right)}-(1-u) H_{n-k, q}^{(s)}(x, y) q^{\left(\begin{array}{c}
k \\
2
\end{array}\right)}(-1)^{k}\right] .
\end{gathered}
$$

Proof. Consider the following identity

$$
\frac{u}{\lambda\left(\lambda e_{p, q}(t)-u\right) e_{p, q}(t)}=\frac{1}{\left(\lambda e_{p, q}(t)-u\right)}-\frac{1}{\lambda e_{p, q}(t)} .
$$

Evaluating the following fraction using above identity, we find

$$
\begin{gathered}
\frac{u(1-u) e_{p, q}\left(x t-\frac{s t^{2}}{2}\right) E_{p, q}(y t)}{\lambda\left(\lambda e_{p, q}(t)-u\right) e_{p, q}(t)}=\frac{(1-u) e_{p, q}\left(x t-\frac{s t^{2}}{2}\right) E_{p, q}(y t)}{\lambda e_{p, q}(t)-u} \\
-\frac{(1-u) e_{p, q}\left(x t-\frac{s t^{2}}{2}\right) E_{p, q}(y t)}{\lambda e_{p, q}(t)} \\
u \sum_{n=0}^{\infty} H_{n}^{(s)}(x, y ; u ; \lambda: p, q) \frac{t^{n}}{[n]_{p, q} !}=\lambda \sum_{n=0}^{\infty} H_{n} H_{n}^{(s)}(x, y ; u ; \lambda: p, q) \frac{t^{n}}{[n]_{p, q} !} \sum_{k=0}^{\infty} p^{\left(\begin{array}{c}
k \\
2
\end{array}\right)} \frac{t^{k}}{[k]_{p, q} !} \\
-(1-u) \lambda^{-1} \sum_{n=0}^{\infty} H_{n}^{(s)}(x, y: p, q) \frac{t^{n}}{[n]_{p, q} !} \sum_{k=0}^{\infty} q^{\left(\begin{array}{c}
k \\
2
\end{array}\right)(-1)^{k} \frac{t^{k}}{[k]_{p, q} !} .}
\end{gathered}
$$

Applying the Cauchy product rule in the above equation and then equating the coefficients of like powers of $t$ in both sides of the resultant equation, assertion (3.2) follows.

Theorem 3.3. The following relation for the $(p, q)$-Hermite based Apostol type Frobenius-Euler polynomials ${ }_{H} H_{n}^{(\alpha, s)}(x, y ; u ; \lambda: p, q)$ of order $\alpha$ holds true:

$$
\begin{gathered}
{ }_{H} H_{n}^{(\alpha, s)}(x, y ; u ; \lambda: p, q)=\frac{1}{1-u} \sum_{k=0}^{n}\left(\begin{array}{l}
n \\
k
\end{array}\right)_{p, q}\left[\lambda H_{n-k}(1, y ; u ; \lambda: p, q)_{H} H_{k}^{(\alpha, s)}(x, 0 ; u ; \lambda: p, q)\right. \\
\left.-u_{H} H_{n-k}(0, y ; u ; \lambda: p, q)_{H} H_{k}^{(\alpha, s)}(x, 0 ; u ; \lambda: p, q)\right] .
\end{gathered}
$$

Proof. Consider generating function (2.1), we have

$$
\begin{gathered}
\sum_{n=0}^{\infty} H_{n} H_{n}^{(\alpha, s)}(x, y ; u ; \lambda: p, q) \frac{t^{n}}{[n]_{p, q} !} \\
=\left(\frac{1-u}{\lambda e_{p, q}(t)-u}\right)\left(\frac{\lambda e_{p, q}(t)-u}{1-u}\right)\left(\frac{1-u}{\lambda e_{p, q}(t)-u}\right)^{\alpha} e_{p, q}\left(x t-\frac{s t^{2}}{2}\right) E_{p, q}(y t) \\
=\frac{\lambda}{1-u}\left(\frac{1-u}{\lambda e_{p, q}(t)-u}\right) e_{p, q}(t)\left(\frac{1-u}{\lambda e_{p, q}(t)-u}\right)^{\alpha} e_{p, q}\left(x t-\frac{s t^{2}}{2}\right) E_{p, q}(y t) \\
-\frac{u}{1-u}\left(\frac{1-u}{\lambda e_{p, q}(t)-u}\right)\left(\frac{1-u}{\lambda e_{p, q}(t)-u}\right)^{\alpha} e_{p, q}\left(x t-\frac{s t^{2}}{2}\right) E_{p, q}(y t) .
\end{gathered}
$$


Simplifying the above equation and using equations (2.1) and (1.15), we find

$$
\begin{aligned}
= & \frac{\lambda}{1-u} \sum_{n=0}^{\infty} H_{n}(1, y ; u ; \lambda: p, q) \frac{t^{n}}{[n]_{p, q} !} \sum_{k=0}^{\infty} H_{k}^{(\alpha, s)}(x, 0 ; u ; \lambda: p, q) \frac{t^{k}}{[k]_{p, q} !}- \\
& \frac{u}{1-u} \sum_{n=0}^{\infty} H_{n}(0, y ; u ; \lambda: p, q) \frac{t^{n}}{[n]_{p, q} !} \sum_{k=0}^{\infty} H_{k} H_{k}^{(\alpha, s)}(x, 0 ; u ; \lambda: p, q) \frac{t^{k}}{[k]_{p, q} !} .
\end{aligned}
$$

Applying the Cauchy product rule in the above equation and then equating the coefficients of like powers of $t$ in both sides of the resultant equation, assertion (3.3) follows.

Theorem 3.4. The following recurrence relation for the $(p, q)$-Hermite based Apostol type Frobenius-Euler polynomials ${ }_{H} H_{n}^{(\alpha, s)}(x, y ; u ; \lambda: p, q)$ of order $\alpha$ holds true:

$$
\begin{aligned}
{ }_{H} H_{n+1}^{(s)}(x, y ; u ; \lambda \quad: \quad p, q)=-s[n]_{p, q H} H_{n-1}^{(s)}\left(\frac{q}{p} x, \frac{q}{p} y ; u ; \lambda: p, q\right) p^{n-1} \\
+x_{H} H_{n}^{(s)}(x, y ; u ; \lambda \quad: \quad p, q) p^{n}+y_{H} H_{n}^{(s)}\left(\frac{q}{p} x, \frac{q}{p} y ; u ; \lambda: p, q\right) p^{n} \\
-\frac{\lambda}{1-u} \sum_{k=0}^{n}\left(\begin{array}{l}
n \\
k
\end{array}\right)_{p, q} q^{n-k} p^{k} H_{n-k}^{(s)}(x, y ; u ; \lambda: p, q) H_{n}(1,0 ; u ; \lambda: p, q) .
\end{aligned}
$$

Proof. Taking $\alpha=1$ and then applying $(p, q)$-derivative of generating function (2.1), it follows that

$$
\sum_{n=0}^{\infty}{ }_{H} H_{n+1}^{(s)}(x, y ; u ; \lambda: p, q) \frac{t^{n}}{[n]_{p, q} !}=(1-u) D_{p, q ; t}\left[\frac{e_{p, q}\left(x t-\frac{s t^{2}}{2}\right) E_{p, q}(y t)}{\lambda e_{p, q}(t)-u}\right]
$$

which on performing differentiation in left hand side using formula (1.3) yields

$$
\begin{gathered}
\sum_{n=0}^{\infty}{ }_{H} H_{n+1}^{(s)}(x, y ; u ; \lambda: p, q) \frac{t^{n}}{[n]_{p, q} !}=(1-u) \\
\cdot\left[\frac{\left(\lambda e_{p, q}(q t)-u\right) D_{p, q ; t}\left(e_{p, q}(x t) e_{p, q}\left(-\frac{s t^{2}}{2}\right) E_{p, q}(y t)\right)}{\left(\lambda e_{p, q}(p t)-u\right)\left(\lambda e_{p, q}(q t)-u\right)}\right] \\
-(1-u) \frac{e_{p, q}(p x t) E_{p, q}(p y t) e_{p, q}\left(-\frac{s p^{2} t^{2}}{2}\right) D_{p, q ; t}\left(\lambda e_{p, q}(t)-u\right)}{\left(\lambda e_{p, q}(p t)-u\right)\left(\lambda e_{p, q}(q t)-u\right)} \\
=-s\left(\frac{1-u}{\lambda e_{p, q}(p t)-u}\right) e_{p, q}\left(q x t-\frac{s q^{2} t^{2}}{2}\right) E_{p, q}(q y t) t \\
+x\left(\frac{1-u}{\lambda e_{p, q}(p t)-u}\right) e_{p, q}\left(p x t-\frac{s p^{2} t^{2}}{2}\right) E_{p, q}(p y t) \\
+y\left(\frac{1-u}{\lambda e_{p, q}(p t)-u}\right) e_{p, q}\left(q x t-\frac{s q^{2} t^{2}}{2}\right) E_{p, q}(q y t),
\end{gathered}
$$


then

$$
\begin{aligned}
&-\frac{\lambda}{1-u}\left(\frac{1-u}{\lambda e_{p, q}(p t)-u}\right) e_{p, q}\left(p x t-\frac{s p^{2} t^{2}}{2}\right) E_{p, q}(p y t)\left(\frac{1-u}{\lambda e_{p, q}(q t)-u}\right) e_{p, q}(t) \\
&=-s[n]_{p, q} \sum_{n=0}^{\infty} H_{n-1} H_{n-1}^{(s)}\left(\frac{q}{p} x, \frac{q}{p} y ; u ; \lambda: p, q\right) p^{n-1} \frac{t^{n}}{[n]_{p, q} !} \\
&+x \sum_{n=0}^{\infty} H_{n}^{(s)}(x, y ; u ; \lambda:p, q) p^{n} \frac{t^{n}}{[n]_{p, q} !}+y \sum_{n=0}^{\infty} H_{n}^{(s)}\left(\frac{q}{p} x, \frac{q}{p} y ; u ; \lambda: p, q\right) p^{n} \frac{t^{n}}{[n]_{p, q} !} \\
&-\frac{\lambda}{1-u} \sum_{n=0}^{\infty} H_{n} H_{n}^{(s)}(x, y ; u ; \lambda \quad: \quad p, q) q^{n} \frac{t^{n}}{[n]_{p, q} !} \sum_{k=0}^{\infty} H_{n}(1,0 ; u ; \lambda: p, q) p^{k} \frac{t^{k}}{[k]_{p, q} !}
\end{aligned}
$$

which on making use of the Cauchy product rule in the right hand side and comparing the coefficients of $\frac{t^{n}}{n !}$ on both sides of the resultant equation gives recurrence relation (3.4).

Theorem 3.5. The following relation between the $(p, q)$-Hermite based Apostol type Frobenius polynomials ${ }_{H} H_{n}^{(\alpha, s)}(x, y ; u ; \lambda: p, q)$ and $(p, q)$-Apostol type Bernoulli polynomials $B_{n}(x ; \lambda: p, q)$ holds true:

$$
\begin{gathered}
{ }_{H} H_{n}^{(\alpha, s)}(x, y ; u ; \lambda: p, q)=\sum_{k=0}^{n+1}\left(\begin{array}{c}
n+1 \\
k
\end{array}\right)_{p, q}\left(\lambda \sum_{r=0}^{k}\left(\begin{array}{l}
k \\
r
\end{array}\right)_{p, q} B_{k-r}(x ; \lambda: p, q)-B_{k}(x ; \lambda: p, q)\right) \\
\times{ }_{H} H_{n-k+1}^{(\alpha, s)}(0, y ; u ; \lambda: p, q) .
\end{gathered}
$$

Proof. Considering generating function (2.1), we have

$$
\begin{gathered}
\sum_{n=0}^{\infty} H_{n} H_{n}^{(\alpha, s)}(x, y ; u ; \lambda: p, q) \frac{t^{n}}{[n]_{p, q} !}=\left(\frac{1-u}{e_{p, q}(t)-u}\right)^{\alpha} e_{p, q}\left(x t-\frac{s t^{2}}{2}\right) \\
\cdot E_{p, q}(y t)\left(\frac{t}{\lambda e_{p, q}(t)-1}\right)\left(\frac{\lambda e_{p, q}(t)-1}{t}\right) \\
=\frac{1}{t}\left(\lambda \sum_{n=0}^{\infty} H_{n}^{(\alpha, s)}(0, y ; \lambda) \frac{t^{n}}{[n]_{p, q} !} \sum_{k=0}^{\infty} B_{k}(x ; \lambda: p, q) \frac{t^{k}}{[k]_{p, q} !} \sum_{r=0}^{\infty} \frac{t^{r}}{[r]_{p, q} !}\right. \\
\left.-\sum_{n=0}^{\infty} H_{n}^{(\alpha, s)}(0, y ; u ; \lambda: p, q) \frac{t^{n}}{[n]_{p, q} !} \sum_{k=0}^{\infty} B_{k}(x ; \lambda: p, q) \frac{t^{k}}{[k]_{p, q} !}\right) .
\end{gathered}
$$

On equating the coefficients of same powers of $t$ after using Cauchy product rule in (3.6), assertion (3.5) follows.

Theorem 3.6. The following relation between the $(p, q)$-Hermite based Apostol type Frobenius type Eulerian polynomials ${ }_{H} A_{n, q}^{(\alpha, s)}(x, y ; \lambda)$ and Apostol type Euler polynomials $E_{n, q}(x ; \lambda)$ holds true:

$$
\begin{gathered}
{ }_{H} H_{n}^{(\alpha)}(x, y ; u ; \lambda: p, q)=\frac{1}{2} \sum_{k=0}^{n}\left(\begin{array}{l}
n \\
k
\end{array}\right)_{p, q} \\
\times\left(\lambda \sum_{r=0}^{k}\left(\begin{array}{l}
k \\
r
\end{array}\right)_{p, q} E_{k-r}(x ; \lambda: p, q)+E_{k}(x ; \lambda: p, q)\right)_{H} H_{n-k}^{(\alpha, s)}(0, y ; u ; \lambda: p, q) .
\end{gathered}
$$


Proof. Consider generating function (2.1), we have

$$
\begin{gathered}
\sum_{n=0}^{\infty} H_{n} H_{n}^{(\alpha)}(x, y ; u ; \lambda: p, q) \frac{t^{n}}{[n]_{p, q} !} \\
=\left(\frac{1-u}{e_{p, q}(t)-u}\right)^{\alpha} e_{p, q}\left(x t-\frac{s t^{2}}{2}\right) E_{p, q}(y t)\left(\frac{2}{\lambda e_{p, q}(t)+1}\right)\left(\frac{\lambda e_{p, q}(t)+1}{2}\right) \\
=\frac{1}{2}\left(\lambda \sum_{n=0}^{\infty} H_{n}^{(\alpha, s)}(0, y ; u ; \lambda: p, q) \frac{t^{n}}{[n]_{p, q} !} \sum_{k=0}^{\infty} E_{k}(x ; \lambda: p, q) \frac{t^{k}}{[k]_{p, q} !} \sum_{r=0}^{\infty} \frac{t^{r}}{[r]_{p, q} !}\right. \\
\left.+\sum_{n=0}^{\infty} H_{n}^{(\alpha, s)}(0, y ; u ; \lambda: p, q) \frac{t^{n}}{[n]_{p, q} !} \sum_{k=0}^{\infty} E_{k}(x ; u ; \lambda: p, q) \frac{t^{k}}{[k]_{p, q} !}\right) .
\end{gathered}
$$

On equating the coefficients of same powers of $t$ after using Cauchy product rule in (3.8), assertion (3.7) follows.

Theorem 3.7. The following relation between the $(p, q)$-Hermite based Apostol type Frobenius-Euler polynomials ${ }_{H} H_{n}^{(\alpha, s)}(x, y ; u ; \lambda: p, q)$ and Apostol type Genocchi polynomials $G_{n}(x ; \lambda: p, q)$ holds true:

$$
\begin{gathered}
H_{n}^{(\alpha, s)}(x, y ; u ; \lambda: p, q)=\frac{1}{2} \sum_{k=0}^{n+1}\left(\begin{array}{c}
n+1 \\
k
\end{array}\right)_{p, q} \\
\times\left(\lambda \sum_{r=0}^{k}\left(\begin{array}{c}
k \\
r
\end{array}\right)_{p, q} G_{k-r}(x ; \lambda: p, q)+G_{k}(x ; \lambda: p, q)\right){ }_{H} H_{n-k+1}^{(\alpha, s)}(0, y ; u ; \lambda: p, q) .
\end{gathered}
$$

Proof. Consider generating function (2.1), we have

$$
\begin{gathered}
\sum_{n=0}^{\infty} H_{n} H_{n}^{(\alpha, s)}(x, y ; u ; \lambda: p, q) \frac{t^{n}}{[n]_{p, q} !} \\
=\left(\frac{1-u}{\lambda e_{p, q}(t)-u}\right)^{\alpha} e_{p, q}\left(x t-\frac{s t^{2}}{2}\right) E_{p, q}(y t)\left(\frac{2 t}{\lambda e_{p, q}(t)+1}\right)\left(\frac{\lambda e_{p, q}(t)+1}{2 t}\right) \\
=\frac{1}{2 t}\left(\lambda \sum_{n=0}^{\infty} H_{n}^{(\alpha, s)}(0, y ; u ; \lambda: p, q) \frac{t^{n}}{[n]_{p, q} !} \sum_{k=0}^{\infty} G_{k}(x ; \lambda: p, q) \frac{t^{k}}{[k]_{p, q} !} \sum_{r=0}^{\infty} \frac{t^{r}}{[r]_{p, q} !}\right. \\
\left.+\sum_{n=0}^{\infty} H_{n}^{(\alpha, s)}(0, y ; u ; \lambda: p, q) \frac{t^{n}}{[n]_{p, q} !} \sum_{k=0}^{\infty} G_{k}(x ; \lambda: p, q) \frac{t^{k}}{[k]_{p, q} !}\right) .
\end{gathered}
$$

On equating the coefficients of same powers of $t$ after using Cauchy product rule in (3.10), assertion (3.9) follows.

\section{Summation formulae for $(p, q)$-Hermite Based Apostol type Frobenius-Euler polynomials}

In this section, we provide implicit formulae, Stirling numbers of the second kind and some relationships for $(p, q)$-Hermite based Apostol type Frobenius-Euler polynomials of order $\alpha$ related to $(p, q)$-Apostol type Bernoulli polynomials, $(p, q)$-Apostol type Euler polynomials and $(p, q)$-Apostol type Genocchi polynomials. We now begin with the following theorem. 
Theorem 4.1. The following summation formulae for $(p, q)$-Hermite based Apostol type Frobenius-Euler polynomials of order $\alpha$ holds true:

$$
{ }_{H} H_{k+l}^{(\alpha, s)}(z, y ; u ; \lambda: p, q)=\sum_{n, m=0}^{k, l}\left(\begin{array}{c}
l \\
m
\end{array}\right)_{p, q}\left(\begin{array}{l}
k \\
n
\end{array}\right)_{p, q}(z-x)^{n+m}{ }_{H} H_{k+l-n-m}^{(\alpha, s)}(x, y ; u ; \lambda: p, q) .
$$

Proof. We replace $t$ by $t+w$ and rewrite the generating function (2.1) as

$$
\begin{gathered}
\left(\frac{1-u}{\lambda e_{p, q}(t+w)-u}\right)^{\alpha} E_{p, q}(y(t+w)) e_{p, q}\left(\frac{-s(t+u)^{2}}{2}\right) \\
=e_{p, q}(-x(t+w)) \sum_{k, l=0}^{\infty} H_{k+l}^{(\alpha, s)}(x, y ; u ; \lambda: p, q) \frac{t^{k}}{[k]_{p, q} !} \frac{w^{l}}{[l]_{p, q} !}, \quad \text { (see [11-15]). }
\end{gathered}
$$

Replacing $x$ by $z$ in the above equation and equating the resulting equation to the above equation, we get

$$
\begin{gathered}
e_{p, q}((z-x)(t+w)) \sum_{k, l=0}^{\infty} H_{k+l} H_{k, s)}^{(\alpha)}(x, y ; u ; \lambda: p, q) \frac{t^{k}}{[k]_{p, q} !} \frac{w^{l}}{[l]_{p, q} !} \\
=\sum_{k, l=0}^{\infty}{ }_{H} H_{k+l}^{(\alpha, s)}(z, y ; u ; \lambda: p, q) \frac{t^{k}}{[k]_{p, q} !} \frac{w^{l}}{[l]_{p, q} !} .
\end{gathered}
$$

On expanding exponential function (4.3) gives

$$
\begin{gathered}
\sum_{N=0}^{\infty} \frac{[(z-x)(t+w)]^{N}}{[N]_{p, q} !} \sum_{k, l=0}^{\infty}{ }_{H} H_{k+l}^{(\alpha, s)}(x, y ; u ; \lambda: p, q) \frac{t^{k}}{[k]_{p, q} !} \frac{w^{l}}{[l]]_{p, q} !} \\
=\sum_{k, l=0}^{\infty}{ }_{H} H_{k+l}^{(\alpha, s)}(z, y ; u ; \lambda: p, q) \frac{t^{k}}{[k]_{p, q} !} \frac{w^{l}}{[l]]_{p, q} !}
\end{gathered}
$$

which on using formula [19,p.52(2)]

$$
\sum_{N=0}^{\infty} f(N) \frac{(x+y)^{N}}{N !}=\sum_{n, m=0}^{\infty} f(n+m) \frac{x^{n}}{n !} \frac{y^{m}}{m !}
$$

in the left hand side becomes

$$
\begin{aligned}
& \sum_{n, m=0}^{\infty} \frac{(z-x)^{n+m} t^{n} w^{m}}{[n]_{p, q} ![m]_{p, q} !} \sum_{k, l=0}^{\infty}{ }_{H} H_{k+l}^{(\alpha, s)}(x, y ; u ; \lambda: p, q) \frac{t^{k}}{[k]_{p, q} !} \frac{w^{l}}{[l]_{p, q !}} \\
& =\sum_{k, l=0}^{\infty}{ }_{H} H_{k+l}^{(\alpha, s)}(z, y ; u ; \lambda: p, q) \frac{t^{k}}{[k]_{p, q} !} \frac{w^{l}}{[l]]_{p, q} !} .
\end{aligned}
$$

Now replacing $k$ by $k-n$, and $l$ by $l-m$ in the left hand side of (4.6), we get

$$
\begin{gathered}
\sum_{k, l=0}^{\infty} \sum_{n, m=0}^{k, l} \frac{(z-x)^{n+m}}{[n]_{p, q} ![m]_{p, q} !} H_{k+l-n-m}^{(\alpha, s)}(x, y ; u ; \lambda: p, q) \frac{t^{k}}{(k-n)_{p, q} !} \frac{w^{l}}{(l-m)_{p, q} !} \\
=\sum_{k, l=0}^{\infty} H_{k+l} H_{k, l}^{(\alpha)}(z, y ; u ; \lambda: p, q) \frac{t^{k}}{[k]_{p, q} !} \frac{w^{l}}{[l]_{p, q} !} .
\end{gathered}
$$

Finally on equating the coefficients of the like powers of $t$ and $w$ in the above equation, we get the required result.

Remark 4.1. By taking $l=0$ in Eq. (4.1), we immediately deduce the following result. 
Corollary 4.1. The following summation formula for $(p, q)$-Hermite based Apostol type Frobenius-Euler polynomials of order $\alpha$ holds true:

$$
{ }_{H} H_{k+l}^{(\alpha, s)}(z, y ; u ; \lambda: p, q)=\sum_{n=0}^{k}\left(\begin{array}{l}
k \\
n
\end{array}\right)_{p, q}(z-x)^{n}{ }_{H} H_{k-n}^{(\alpha, s)}(x, y ; u ; \lambda: p, q) .
$$

Remark 4.2. On replacing $z$ by $z+x$ and setting $y=0$ in Theorem (4.1), we get the following result involving $(p, q)$-Hermite based Apostol type Frobenius Euler polynomials ${ }_{H} H_{n}^{(\alpha, s)}(x, y ; u ; \lambda: p, q)$ of one variable

$$
{ }_{H} H_{k+l}^{(\alpha, s)}(z+x ; u ; \lambda: p, q)==\sum_{n, m=0}^{k, l}\left(\begin{array}{c}
l \\
m
\end{array}\right)_{p, q}\left(\begin{array}{l}
k \\
n
\end{array}\right)_{p, q} z^{n+m}{ }_{H} H_{k+l-n-m}^{(\alpha, s)}(x ; u ; \lambda: p, q),
$$

whereas by setting $z=0$ in Theorem 4.1, we get another result involving $(p, q)$-Hermite based Apostol type Frobenius Euler polynomials ${ }_{H} H_{n}^{(\alpha, s)}(x, y ; u ; \lambda: p, q)$ of one and two variables

$$
\begin{gathered}
{ }_{H} H_{k+l}^{(\alpha, s)}(y ; u ; \lambda: p, q) \\
=\sum_{n, m=0}^{k, l}\left(\begin{array}{c}
l \\
m
\end{array}\right)_{p, q}\left(\begin{array}{c}
k \\
n
\end{array}\right)_{p, q}(-x)^{n+m}{ }_{H} H_{k+l-n-m}^{(\alpha, s)}(x, y ; u ; \lambda: p, q) .
\end{gathered}
$$

Theorem 4.2. The following summation formulae for $(p, q)$-Hermite based Apostol type Frobenius Euler polynomials ${ }_{H} H_{n}^{(\alpha, s)}(x, y ; u ; \lambda: p, q)$ of order $\alpha$ holds true:

$$
{ }_{H} H_{n}^{(\alpha+1, s)}(x, y ; u ; \lambda: p, q)=\sum_{m=0}^{n}\left(\begin{array}{c}
n \\
m
\end{array}\right)_{p, q} H_{n-m}(u ; \lambda: p, q)_{H} H_{m}^{(\alpha, s)}(x, y ; u ; \lambda: p, q) .
$$

Proof. From (2.1), we have

$$
\begin{aligned}
& \frac{1-u}{\lambda e_{p, q}(t)-u}\left(\frac{1-u}{\lambda e_{p, q}(t)-u}\right)^{\alpha} e_{p, q}\left(x t-\frac{s t^{2}}{2}\right) E_{p, q}(y t) \\
= & \frac{1-u}{\lambda e_{p, q}(t)-u} \sum_{m=0}^{\infty} H_{m}^{(\alpha, s)}(x, y ; u ; \lambda: p, q) \frac{t^{m}}{[m]_{p, q} !} \\
= & \frac{1-u}{\lambda e_{p, q}(t)-u} \sum_{m=0}^{\infty} H_{m}^{(\alpha)}(x, y ; u ; \lambda: p, q) \frac{t^{m}}{[m]_{p, q} !},
\end{aligned}
$$

then,

$$
\sum_{n=0}^{\infty}{ }_{H} H_{n}^{(\alpha+1, s)}(x, y ; u ; \lambda: p, q) \frac{t^{n}}{[n]_{p, q} !}=\sum_{n=0}^{\infty} H_{n}(u ; \lambda: p, q) \frac{t^{n}}{[n]_{p, q} !} \sum_{m=0}^{\infty}{ }_{H} H_{m}^{(\alpha, s)}(x, y ; u ; \lambda: p, q) \frac{t^{m}}{[m]_{p, q} !} .
$$

Now replacing $n$ by $n-m$ and equating the coefficients of $t^{n}$ leads to formula (4.11).

Theorem 4.3. The following summation formulae for $(p, q)$-Hermite based Apostol type Frobenius Euler polynomials ${ }_{H} H_{n}^{(\alpha, s)}(x, y ; u ; \lambda: p, q)$ of order $\alpha$ holds true:

$$
{ }_{H} H_{n}^{(\alpha, s)}(x+1, y ; u ; \lambda: p, q)=\sum_{k=0}^{n}\left(\begin{array}{l}
n \\
k
\end{array}\right)_{p, q}{ }_{H} H_{k}^{(\alpha, s)}(x, y ; u ; \lambda: p, q) .
$$


Proof. Using definition (2.1), we have

$$
\begin{aligned}
& \sum_{n=0}^{\infty}{ }_{H} H_{n}^{(\alpha, s)}(x+1, y ; u ; \lambda: p, q) \frac{t^{n}}{[n]_{p, q} !}-\sum_{n=0}^{\infty}{ }_{H} H_{n}^{(\alpha, s)}(x, y ; u ; \lambda) \frac{t^{n}}{[n]_{p, q} !} \\
& =\left(\frac{1-u}{\lambda e_{p, q}(t)-u}\right)^{\alpha} e_{p, q}\left(x t-\frac{s t^{2}}{2}\right) E_{p, q}(y t)\left(e_{p, q}(t)-1\right) \\
& =\left(\sum_{k=0}^{\infty} H_{k}^{(\alpha, s)}(x, y ; u ; \lambda: p, q) \frac{t^{k}}{[k]_{p, q} !}\right)\left(\sum_{n=0}^{\infty} \frac{t^{n}}{[n]_{p, q} !}\right) \\
& -\sum_{n=0}^{\infty} H_{n}^{(\alpha, s)}(x, y ; u ; \lambda: p, q) \frac{t^{n}}{[n]_{p, q} !} \\
& =\sum_{n=0}^{\infty} \sum_{k=0}^{n}{ }_{H} H_{k}^{(\alpha, s)}(x, y ; u ; \lambda: p, q) \frac{t^{n}}{(n-k)_{p, q} !}-\sum_{n=0}^{\infty}{ }_{H} H_{n}^{(\alpha, s)}(x, y ; u ; \lambda: p, q) \frac{t^{n}}{[n]_{p, q} !} .
\end{aligned}
$$

Finally, equating the coefficients of the like powers of $t^{n}$, we get (4.12).

Theorem 4.4. Let $\alpha$ and $\gamma$ be nonnegative integers. There is a relationship between the numbers $S(n, k, \lambda$ : $p, q)$ and the $(p, q)$-Hermite based Apostol type Frobenius-Euler polynomials ${ }_{H} H_{n}^{(\alpha, s)}(x, y ; u ; \lambda: p, q)$ of order $\alpha$ holds true:

$$
\begin{gathered}
\alpha ! \sum_{l=0}^{n}\left(\begin{array}{l}
n \\
l
\end{array}\right)_{p, q}{ }_{H} H_{n-l}^{(\alpha, s)}(x, y ; u ; \lambda: p, q) S\left(l, \alpha, \frac{\lambda}{u}: p, q\right)=\left(\frac{1-u}{u}\right)^{\alpha} H_{n}^{(s)}(x, y: p, q) . \\
{ }_{H} H_{n}^{(\alpha-\gamma, s)}(x, y ; u ; \lambda: p, q)=\gamma !\left(\frac{u}{1-u}\right)^{\gamma} \alpha ! \\
\sum_{l=0}^{n}\left(\begin{array}{c}
n \\
l
\end{array}\right)_{p, q}{ }_{H} H_{n-l}^{(\alpha, s)}(x, y ; u ; \lambda: p, q) S\left(l, \alpha, \frac{\lambda}{u}: p, q\right) .
\end{gathered}
$$

Proof. By using generating function (2.1), we have

$$
\begin{gathered}
\sum_{n=0}^{\infty} H_{n} H_{n}^{(\alpha, s)}(x, y ; u ; \lambda: p, q) \frac{t^{n}}{[n]_{p, q} !}=\left(\frac{1-u}{\lambda e_{p, q}(t)-u}\right)^{\alpha} e_{p, q}\left(x t-\frac{s t^{2}}{1+q}\right) E_{p, q}(y t) \\
\left(\lambda e_{p, q}(t)-u\right)^{\alpha} \sum_{n=0}^{\infty} H_{n} H_{n}^{(\alpha, s)}(x, y ; u ; \lambda: p, q) \frac{t^{n}}{[n]_{p, q} !}=(1-u)^{\alpha} \sum_{n=0}^{\infty} H_{n}^{(s)}(x, y: p, q) \frac{t^{n}}{[n]_{p, q} !} \\
\alpha ! \frac{\left(\frac{\lambda}{u} e_{p, q}(t)-1\right)^{\alpha}}{\alpha !} \sum_{n=0}^{\infty} H_{n} H_{n}^{(\alpha, s)}(x, y ; u ; \lambda: p, q) \frac{t^{n}}{[n]_{p, q} !}=\left(\frac{1-u}{u}\right)^{\alpha} \sum_{n=0}^{\infty} H_{n}^{(s)}(x, y: p, q) \frac{t^{n}}{[n]_{p, q} !},
\end{gathered}
$$

which on rearranging the terms using equation (2.3) and the following expansion as:

$$
\frac{\left(\lambda e^{t}-k\right)^{\alpha}}{k !}=\sum_{n=0}^{\infty} S(n, k, \lambda) \frac{t^{n}}{n !}, \quad(\text { see }[17]),
$$

and then

$$
\begin{gathered}
\alpha ! \sum_{n=0}^{\infty} H_{n} H_{n}^{(\alpha, s)}(x, y ; u ; \lambda: p, q) \frac{t^{n}}{[n]_{p, q} !} \sum_{l=0}^{\infty} S\left(l, \alpha, \frac{\lambda}{u}: p, q\right) \frac{t^{l}}{[l]_{p, q} !} \\
=\left(\frac{1-u}{u}\right)^{\alpha} \sum_{n=0}^{\infty} H_{n}^{(s)}(x, y: p, q) \frac{t^{n}}{[n]_{p, q} !}
\end{gathered}
$$

which on rearranging the summation and then simplifying the resultant equation yields relation (4.13). 
Again, we consider the following arrangement of generating function (2.1) as:

$$
\begin{gathered}
\sum_{n=0}^{\infty} H_{n} H_{n}^{(\alpha-\gamma, s)}(x, y ; u ; \lambda: p, q) \frac{t^{n}}{[n]_{p, q} !} \\
=\left(\frac{1-u}{\lambda e_{p, q}(t)-u}\right)^{\alpha} e_{p, q}\left(x t-\frac{s t^{2}}{1+q}\right) E_{p, q}(y t)\left(\frac{u}{1-u}\right)^{\gamma} \gamma ! \frac{\left(\frac{\lambda}{u} e_{p, q}(t)-1\right)^{\gamma}}{\gamma !},
\end{gathered}
$$

which on use of equations (4.15) and (2.1) and applying Cauchy product rule and then canceling the same powers of $t$ in resultantly equation yields relation (4.14).

Theorem 4.5. The following relationship holds true:

$$
\begin{gathered}
{ }_{H} H_{n}^{(\alpha, s)}(x, y ; u ; \lambda: p, q) \\
=\sum_{j=0}^{n} \sum_{l=j}^{n}\left(\begin{array}{c}
\alpha+j-1 \\
j
\end{array}\right)_{p, q} j !\left(\begin{array}{c}
n \\
l
\end{array}\right)_{p, q}(1-u)^{-j} S_{2}(l, j ; \lambda: p, q)(\lambda-1)^{l-j} H_{n-l}^{(s)}(x, y ; p, q) .
\end{gathered}
$$

Proof. From (2.1), we have

$$
\begin{gathered}
\sum_{n=0}^{\infty} H_{n}^{(\alpha, s)}(x, y ; u ; \lambda: p, q) \frac{t^{n}}{[n]_{p, q} !}=\left(\frac{1-u}{\lambda e_{p, q}(t)-u}\right)^{\alpha} e_{p, q}\left(x t-\frac{s t^{2}}{2}\right) E_{p, q}(y t) \\
=e_{p, q}\left(x t-\frac{s t^{2}}{2}\right) E_{p, q}(y t)\left(1+\frac{\lambda e_{p, q}(t)-1}{1-u}\right)^{-\alpha} \\
=\sum_{j=0}^{\infty}\left(\begin{array}{c}
-\alpha \\
j
\end{array}\right)_{p, q}\left(\frac{\lambda e_{p, q}(t)-1}{1-u}\right)^{j} \sum_{n=0}^{\infty} H_{n}^{s}(x, y: p, q) \frac{t^{n}}{[n]_{p, q} !} \\
=\sum_{n=0}^{\infty}\left(\sum_{j=0}^{n} \sum_{l=j}^{n}\left(\begin{array}{c}
\alpha+j-1 \\
j
\end{array}\right)_{p, q} j !\left(\begin{array}{c}
n \\
l
\end{array}\right)_{p, q}(1-u)^{-j} S_{2}(l, j ; \lambda: p, q) H_{n-l}^{(s)}(x, y: p, q)\right) \frac{t^{n}}{[n]_{p, q} !} .
\end{gathered}
$$

On comparing the coefficients of $t^{n}$ in both sides, we get (4.17).

Theorem 4.6. The following relationship holds true:

$$
{ }_{H} H_{n}^{(\alpha, s)}(x, y ; u ; \lambda: p, q)=\sum_{k=0}^{n}(\alpha){ }_{k}(u-1)^{-k} S_{n}^{(k, s)}(x, y ; \lambda: p, q) .
$$

Proof. By using generating function (2.1), we have

$$
\begin{aligned}
\sum_{n=0}^{\infty}{ }_{H} H_{n}^{(\alpha, s)}(x, y ; u ; \lambda & : \quad p, q) \frac{t^{n}}{[n]_{p, q} !}=\left(\frac{1-u}{\lambda e_{p, q}(t)-u}\right)^{\alpha} e_{p, q}\left(x t-\frac{s t^{2}}{2}\right) E_{p, q}(y t) \\
& =e_{p, q}\left(x t-\frac{s t^{2}}{2}\right) E_{p, q}(y t)\left(1-\frac{\lambda e_{p, q}(t)-1}{u-1}\right)^{-\alpha} \\
& =e_{p, q}\left(x t-\frac{s t^{2}}{2}\right) E_{p, q}(y t)\left(\frac{u-\lambda e_{p, q}(t)}{u-1}\right)^{-\alpha} \\
& =e_{p, q}\left(x t-\frac{s t^{2}}{2}\right) E_{p, q}(y t) \sum_{k=0}^{\infty}(\alpha)_{k} \frac{1}{[k]_{p, q} !}\left(\frac{\lambda e_{p, q}(t)-1}{u-1}\right)^{k} \\
& =\sum_{k=0}^{\infty}(\alpha)_{k} \frac{1}{(u-1)^{k}}\left(\sum_{n=k}^{\infty} S_{n}^{(k, s)}(x, y ; \lambda: p, q) \frac{t^{n}}{[n]_{p, q} !}\right)
\end{aligned}
$$


Since $(1-z)^{-a}=\sum_{n=0}^{\infty}(a)_{n} \frac{z^{n}}{n !}$, we have

$$
\sum_{n=0}^{\infty} H_{n}^{(\alpha, s)}(x, y ; u ; \lambda: p, q) \frac{t^{n}}{[n]_{p, q} !}=\sum_{n=0}^{\infty}\left(\sum_{k=0}^{n}(\alpha)_{k}(u-1)^{-k} S_{n}^{(k, s)}(x, y ; \lambda: p, q)\right) \frac{t^{n}}{[n]_{p, q} !} .
$$

Comparing the coefficients of $t^{n}$ in both sides, we arrive at the rapiered result (4.18).

Competing Interests: The authors declare that there is not any conflict of interests regarding the publication of this manuscript.

\section{References}

[1] Carlitz, L., Eulerian numbers and polynomials, Mat. Mag., 32 (1959), 164-171.

[2] Cheon, G. S., A note on the Bernoulli and Euler polynomials, Appl. Math. Lett., 16 (2003) 365-368.

[3] Gupta, V., ( $p, q)$-Baskakov-Kontorovich operators, Appl. Math. Inf. Sci., 10(4) (2016), 1551-1556.

[4] Gupta, V., Aral, A., Bernstein Durrmeyer operators and based on two parameters, Facta Universitatis (Nis), Ser. Math. Inform., 31(1) (2016), 79-95.

[5] Duran, U., Acikgoz, M., Araci, S, On higher order $(p, q)$-Frobenius-Euler polynomials, TWMS J. Pure. Appl. Math., 8(2) (2017), 198-208.

[6] Duran, U., Acikgoz, M., Apostol type $(p, q)$-Frobenious-Euler polynomials and numbers, Kragujevac J. Math., 42(4) (2018), 555-567.

[7] Duran, U., Acikgoz, M., Apostol type $(p, q)$-Bernoulli, $(p, q)$-Euler and $(p, q)$-Genocchi polynomials and numbers, Comput. Appl. Math., 8(1) (2017), 7-30.

[8] Duran, U., Acikgoz, M., Araci, S, On $(p, q)$-Bernoulli, $(p, q)$-Euler and $(p, q)$-Genocchi polynomials, J. Comput, Theor. Nanosci., 13(2016), 7833-7908.

[9] Kurt, V., Simsek, Y., On the generalized Apostol type Frobenius Euler polynomials, Advances in difference equations, (2013)(1) (2013), '1-9.

[10] Kurt, B, A note on the Apostol type $q$-Frobenius Euler polynomials and generalizations of the Srivastava-Pinter addition theorems, Filomat, 30(1) (2016), 65-72.

[11] Khan, W. A, Araci, S., Acikgoz, M., Haroon, H., A new class of partially degenerate HermiteGenocchi polynomials, J. Nonlinear Sci. Appl., 10 (2017), 5072-5081.

[12] Khan, W. A., Some properties of the Generalized Apostol type Hermite based polynomials, Kyungpook Math. J., 55 (2015), 597-614.

[13] Khan, W. A., Haroon, H., Some symmetric identities for the generalized Bernoulli, Euler and Genocchi polynomials associated with Hermite polynomials, Springer Plus, 5 (2016), 1-21.

[14] Pathan, M. A., Khan, W. A., Some implicit summation formulas and symmetric identities for the generalized Hermite-Bernoulli polynomials, Mediterr. J. Math., 12 (2015), 679-695.

[15] Pathan, M. A., Khan, W. A., A new class of generalized polynomials associated with Hermite and Euler polynomials, Mediterr. J. Math., 13(3) (2016), 913-928.

[16] Ryoo, C. S., A note on the Frobenius Euler polynomials, Proc. Jangjeon Math. Soc., 14(4) (2011), 495-501.

[17] Simsek, Y., Generating functions for generalized Stirling type numbers, Array type polynomials, Eulerian type polynomials and their applications, Fixed Point The. Appl., Doi: 1186/1687-18122013-87, (2013).

[18] Simsek, Y, Generating functions for $q$-Apostol type Frobenius-Euler numbers and polynomials, Axioms, 1 (2012), 395-403.

[19] Srivastava, H. M, and Manocha, H. L, A treatise on generating functions, Ellis Horwood Limited. Co. New York, 1984. 
[20] Sadjang, P. N., On the fundamental theorem of $(p, q)$-calculus and some $(p, q)$-Taylor formulas, Results Math., (To appear).

[21] Sadjang, P. N., On $(p, q)$-Appell polynomials, arXiv:1712.01324v1[math.CA] 8 Nov 2017.

[22] Srivastava, H. M., Pintér, A. Remarks on some relationships between the Bernoulli and Euler polynomials, Appl. Math. Lett., 17 (2004), 375-380. 